\title{
Influence of Relativistic Effects on the Contact Formation of Transition Metals
}

\author{
M. R. Calvo, ${ }^{1,2,3,{ }^{*}}$ C. Sabater, ${ }^{1, \dagger}$ W. Dednam, ${ }^{1,4}$ E. B. Lombardi, ${ }^{5}$ M. J. Caturla, ${ }^{1}$ and C. Untiedt ${ }^{1, \$}$ \\ ${ }^{1}$ Departamento de Física Aplicada and Unidad asociada CSIC, Universidad de Alicante, \\ Campus de San Vicente del Raspeig, E-03690 Alicante, Spain \\ ${ }^{2}$ Ikerbasque, Basque Foundation for Science, 48013 Bilbao, Spain \\ ${ }^{3}$ CIC nanoGUNE, 20018 Donostia-San Sebastian, Spain \\ ${ }^{4}$ Department of Physics, University of South Africa, Science Campus, Florida Park, Johannesburg 1710, South Africa \\ ${ }^{5}$ College of Graduate Studies, University of South Africa, Pretoria 0003, South Africa
}

(Received 26 September 2017; published 16 February 2018)

\begin{abstract}
Our analysis of the contact formation processes undergone by $\mathrm{Au}, \mathrm{Ag}$, and $\mathrm{Cu}$ nanojunctions reveals that the distance at which the two closest atoms on a pair of opposing electrodes jump into contact is, on average, 2 times longer for $\mathrm{Au}$ than either $\mathrm{Ag}$ or $\mathrm{Cu}$. This suggests the existence of a longer-range interaction between those two atoms in the case of $\mathrm{Au}$, a result of the significant relativistic energy contributions to the electronic structure of this metal, as confirmed by $a b$ initio calculations. Once in the contact regime, the differences between $\mathrm{Au}, \mathrm{Ag}$, and $\mathrm{Cu}$ are subtle, and the conductance of single-atom contacts for metals of similar chemical valence is mostly determined by geometry and coordination.
\end{abstract}

DOI: 10.1103/PhysRevLett.120.076802

As the atomic mass increases, relativistic effects come into play that modify the electronic structure and thus determine the properties of heavy-element crystals and compounds (for a review see, e.g., Ref. [1]). As a consequence of this, $5 d$ metals differ markedly from their $4 d$ counterparts: examples include $\mathrm{Hg}$ being liquid at standard conditions [2] and the golden luster of bulk $\mathrm{Au}$ [1]. In $5 d$ transition metals, relativistic energy contributions result in a contraction of the outer $6 s$ shell, accompanied by the expansion of the filled $5 d$ orbitals [1], producing an enhancement in the $s-d$ hybridization of the valence orbitals. A first consequence of this is the lattice constant for Au bulk crystals being slightly smaller than for $\mathrm{Ag}$, while based on atomic radii only, it would be expected to increase [1,3], with $\mathrm{Au}$ larger than Ag. Relativistic effects also have a strong influence on the bonding properties of $5 d$ atoms and thus determine the chemistry of the $5 d$ elements [1,4], as well as many physical properties of their bulk crystals. Au exhibits distinct mechanical and structural properties such as a larger bulk moduli and cohesive energies than $\mathrm{Ag}$ [3]. This effect is further amplified in low-coordination structures, where it gives rise to phenomena such as surface reconstruction $[5,6]$. A similar origin is attributed to the formation of monoatomic chains during the rupture of $\mathrm{Au}, \mathrm{Pt}$, and $\mathrm{Ir}$ nanostructures [7], in agreement with expected enhanced $s-d$ hybridization of the valence orbitals in these one-dimensional structures [8-11].

The process of contact formation at the nanoscopic scale has been the object of extensive study in the context of single-atom metallic junctions $[12,13]$. The influence of geometry and coordination on both the contact formation process as well as the characteristics of the resulting structures, is well established for a variety of metals [14-17]. In particular, for $\mathrm{Au}, \mathrm{Ag}$, and $\mathrm{Cu}$, it is well known that first contact in low-coordination geometries is invariably accompanied by an abrupt jump [14,18], upon which a single or double atomic contact is normally found to have formed immediately afterward.

In this work, the formation of thousands of atomic contacts made of pure $\mathrm{Au}, \mathrm{Ag}$, and $\mathrm{Cu}$, is studied and compared. In the contact regime, and in agreement with previous works, the conductance is mainly determined by the valence of the metal [19] and the exact geometry of the contacts $[12,14,15,17]$. In the tunneling regime, on the contrary, we find that there is a remarkable difference between $\mathrm{Au}$, on one hand, and $\mathrm{Ag}$ and $\mathrm{Cu}$, on the other, in terms of the distance from which jump to contact starts, which we show here to be a consequence of the larger relativistic effects in the electronic structure of Au.

Our atomic contacts are fabricated by cyclic loading of two electrode probes made of the same high-purity (99.999\%) metal, $\mathrm{Au}, \mathrm{Ag}$, or $\mathrm{Cu}$, under cryogenic vacuum at $4.2 \mathrm{~K}$. The electrical conductance (obtained as the current divided by the applied voltage of $100 \mathrm{mV}$ ) is recorded while the two electrodes are carefully brought into contact in a scanning tunneling microscope (STM) setup. The process is described in detail in previous works [14,15]. Traces of conductance, such as the one shown in Fig. 1, can be obtained in this way. When the atomic-sized electrodes are close enough but not yet in contact, electrons may tunnel from one to the other. In this tunneling regime, the conductance increases exponentially with decreasing distance between the leads. This increase in conductance remains smooth until a sudden jump occurs, and a plateau at around the value of one 


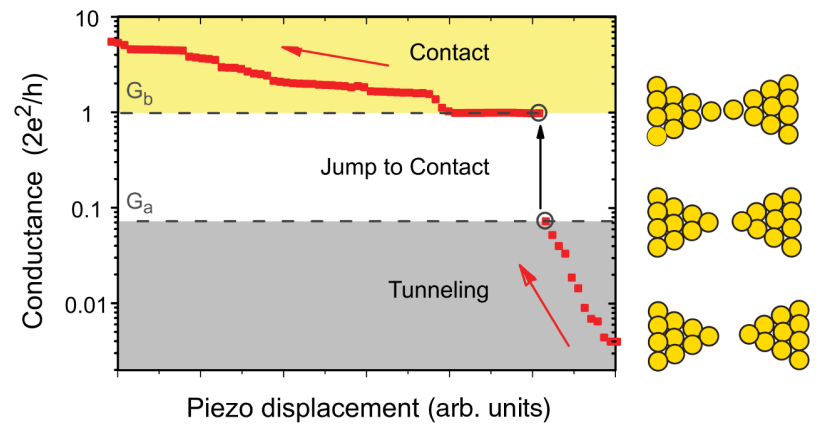

FIG. 1. A trace of conductance recorded during the formation of a gold contact in a STM setup at $4.2 \mathrm{~K}$. As the electrodes approach each other, an initial exponential increase in conductance is followed by a jump into contact, as indicated by the vertical black arrow. Conductance values before and after contact formation are marked and labeled as $G_{a}$ and $G_{b}$, respectively. On the right-hand side, from bottom to top, different stages of the contact process are illustrated.

quantum of conductance $G_{0}=2 e^{2} / h$ appears, indicating the formation of a monatomic contact [12].

For each contact-formation trace, we search for the largest jump in conductance between two consecutive points. Thus, two conductance values are recorded, $G_{a}$, from which the jump occurs, and $G_{b}$, the final value immediately after the jump, labeled accordingly in Fig. 1. Following the analysis introduced by Untiedt et al. [14], we construct density plots from this set of data pairs $\left(G_{a}, G_{b}\right)$. The top row of panels in Fig. 2 represents such density plots, compiled from more than 2000 contacts formed by $\mathrm{Au}, \mathrm{Ag}$, and $\mathrm{Cu}$, respectively. Density maxima appear at the most probable values of $\left(G_{a}, G_{b}\right)$ from and to which the conductance jump occurs.

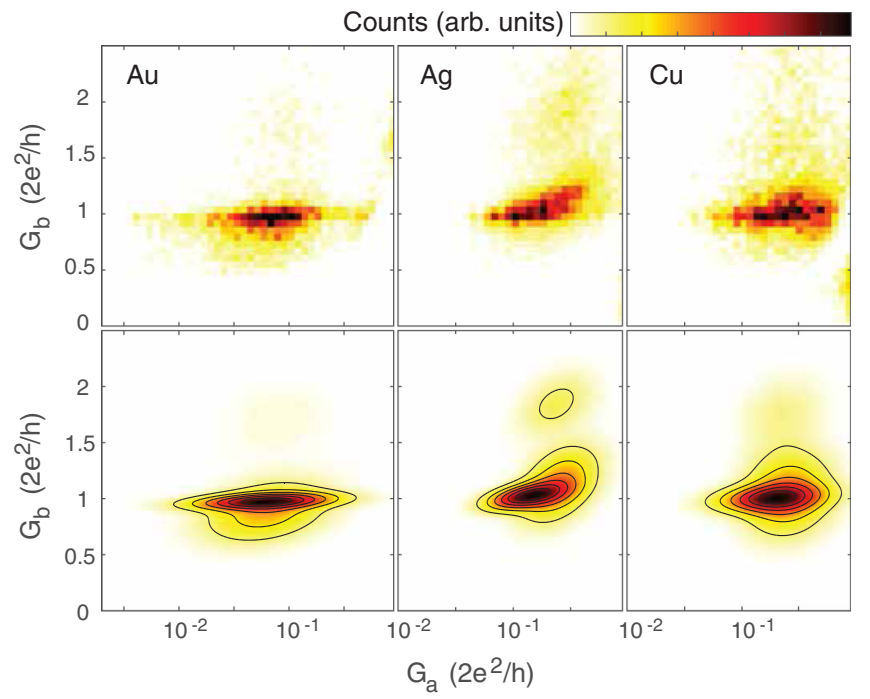

FIG. 2. Upper panels: Density plots of jump to contact parameters extracted from more than 2000 contact formation traces for $\mathrm{Au}, \mathrm{Ag}$, and $\mathrm{Cu}$ (labeled correspondingly). Bottom panels: Evaluation of the results from fitting the data in the upper panels to a sum of three bivariate normal distributions.
The results for Au, presented in the left-hand panels of Fig. 2, are similar to those reported in previous works $[14,15,17]$, except that here we have plotted the $G_{a}$ data on a logarithmic scale. This, which could be seen as just a subtle change, enables a new and improved analysis of these data. First, the log scale conveys a more physical interpretation of our results, since $\log \left(G_{a}\right)$ [20] is directly proportional to the distance between electrodes. Note that the conductance in the tunneling regime depends exponentially on the distance between the electrodes as $G \simeq$ $K e^{-(\sqrt{2 m \phi} / h) d}$, where $K$ is a proportionality constant which depends on the area and density of states at the Fermi level of the electrodes, $m$ corresponds to the electron mass, and $\phi$ is the metal work function. Therefore, a change in conductance of 1 order of magnitude corresponds roughly to a variation in distance of $1 \AA$.

Moreover, Fig. 2 reveals relevant information on the statistical distribution of data, unaccessible before. When plotted on a $G_{a}$ linear scale as in Ref. [14], density plots exhibit a triangular shape, allowing only for a rough identification of distributions and their most probable values on $G_{a}$. In contrast, on a logarithmic scale (Fig. 2), density plots resemble normal distributions in both $G_{b}$ and $\log \left(G_{a}\right)$. In fact, the density maximum around the quantum of conductance can be modeled as the superposition of two distributions. A third maximum, associated with a lower number of counts, can also be observed at higher values of $G_{b}$. Hence, our data can be fitted to the sum of three bivariate normal distributions (see Ref. [21] for details), and thus allowing for a more precise identification of distributions as well as their mean and standard deviation values. Fit results are shown in the lower panels of Fig. 2 and graphically summarized in Fig. 3. In a related work (see Ref. [21]), we show how these three

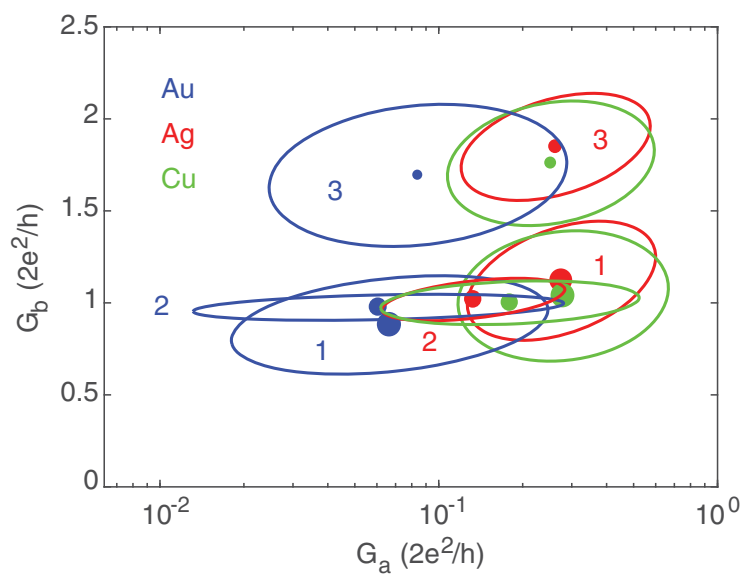

FIG. 3. Graphical summary of the fitting results used to compare the metals. Dots mark the mean value of the distribution, the size of which is proportional to the ratio of occurrence of each configuration, and the ellipse contour encloses the equivalent of 1 standard deviation for each bivariate distribution; in other words, the ellipse encloses $68 \%$ of the data. 
maxima correspond to three first-contact configuration categories, namely, monomers, dimers, and double contacts, in agreement with previous works $[14,15,18]$. However, in contrast to those works, the distributions corresponding to monomers and dimers can now be clearly disentangled. Furthermore, in Ref. [21] we also present a more precise identification of contact geometry and conductance based on our analysis.

A comparison of the results for $\mathrm{Au}, \mathrm{Ag}$, and $\mathrm{Cu}$ contacts reveals a striking difference between $\mathrm{Au}$ and the other two metals, in the distribution of $G_{a}$ values. As listed in Table I and clearly visible in Fig. 3, the mean value of the distance over which Au jumps into contact [proportional to $\log \left(G_{a}\right)$ ] is much larger in magnitude, for all three of its associated distributions. Assuming that in tunneling an increase of an order of magnitude in conductance corresponds approximately to a change of $1 \AA$ in distance, jump to contact for gold occurs at distances up to $\sim 0.5 \AA$ larger than $\mathrm{Ag}$ and $\mathrm{Cu}$, with a broader distribution. This compares favorably with the mean binding lengths calculated for $\mathrm{Au}$ and $\mathrm{Ag}$ junctions from experimental force-extension curves in Ref. [22], which in the case of $\mathrm{Au}$ is $\sim 0.8 \AA$ longer. At the same time, the conductance at first contact exhibited by $\mathrm{Au}$ is slightly smaller than for $\mathrm{Ag}$ and $\mathrm{Cu}$, which, in turn, exhibit similar values.

All of the above can be understood in terms of a longerrange interatomic potential felt by the atoms on opposing $\mathrm{Au}$ electrodes, as compared to electrodes made of $\mathrm{Ag}$ or $\mathrm{Cu}$. In the case of gold, this interaction manifests much sooner, as the force required relative to the bulk elasticity to provoke the jump to contact. The stronger interaction also implies a smoother variation of the interatomic potential as a function of the separation between the Au electrodes, which explains the broader distribution in $G_{a}$ values that is observed. Finally, the fact that Au jumps to contact earlier produces strained structures exhibiting a somewhat lower conductance, which in the case of a dimeric configuration gives way to the narrower distribution of $G_{b}$ values seen in Fig. 3 .

Hence, the longer-range interaction would then seem to explain all the observed features of gold. Since $\mathrm{Au}, \mathrm{Ag}$, and $\mathrm{Cu}$ share very similar electronic configurations, one can

TABLE I. Mean values of $\log \left(G_{a} / G_{0}\right)$ ( \pm standard deviation) values extracted from the fitting of $\mathrm{Au}, \mathrm{Ag}$ and $\mathrm{Cu}$ density plots to three bivariate distributions, labeled as 1,2 and 3 in Fig. 3 and associated respectively to monomer, dimer and double bond geometries [14,21]. $G_{0}=2 e^{2} / h$ denotes here a quantum of conductance.

\begin{tabular}{cccc}
\hline \hline \multicolumn{4}{c}{$\boldsymbol{\mu}_{\log \left(\boldsymbol{G}_{a} / \boldsymbol{G}_{0}\right)}\left( \pm \boldsymbol{\sigma}_{\log \left(\boldsymbol{G}_{a} / \boldsymbol{G}_{0}\right)}\right)$} \\
\hline & $\mathrm{Au}$ & $\mathrm{Ag}$ & $\mathrm{Cu}$ \\
\hline $\mathbf{1}$ & $-1.2 \pm 0.4$ & $-0.6 \pm 0.2$ & $-0.6 \pm 0.2$ \\
$\mathbf{2}$ & $-1.2 \pm 0.4$ & $-0.9 \pm 0.2$ & $-0.8 \pm 0.3$ \\
$\mathbf{3}$ & $-1.1 \pm 0.4$ & $-0.6 \pm 0.2$ & $-0.6 \pm 0.2$ \\
\hline \hline
\end{tabular}

expect the long-range interaction here to originate from relativistic effects, as these are responsible for other similar physical properties in which $\mathrm{Au}$ differs from $\mathrm{Ag}$ and $\mathrm{Cu}$ [3,5-7], as previously explained.

To test the above hypothesis, we have performed scalar relativistic and nonrelativistic total-energy density functional theory (DFT) calculations on infinite monatomic chains of gold and silver [9]. For this, we have employed the planewave DFT code CASTEP [23], explicitly including or excluding scalar relativistic interactions. We make use of on-the-flygenerated (OTFG) pseudopotentials [24] (benchmarked against fully converged all-electron DFT calculations, with an error of $0.5 \mathrm{meV} /$ atom obtained by the methods described in Ref. [25]). The (scalar) relativistic treatment is at the level of the Koelling-Harmon approximation of the Dirac equation [26], which, with the exception of spin-orbit coupling (SOC), retains all other relativistic kinematic effects such as mass-velocity, Darwin, and higherorder terms. Since monatomic chains and atomic point contacts made of gold do not appear to exhibit significant local magnetic order [27], we have neglected SOC in our calculations. As the exchange-correlation functional, we have used the generalized gradient approximation by Perdew-Burke-Ernzerhof [28]. We used the TkatchenkoScheffler dispersion-correction scheme to take van der Waals interactions between atoms into account. van der Waals interactions more accurately describe long-range interaction tails between atoms and molecules in vacuum slabs [29], and even produce improved agreement with experimental bulk properties [30]. We have also used the plane-wave cutoff in Ref. [31] for gold, $400 \mathrm{eV}$, while silver required a larger value, $600 \mathrm{eV}$. Convergence was checked with respect to the plane-wave cutoff, with total energies converged to within $5 \times 10^{-7} \mathrm{eV} /$ atom. A total of 24 irreducible $k$ points were used to sample reciprocal space in our calculations. Convergence was also checked for $k$ points by gradually increasing the size of the Monckhorst-Pack grid automatically generated by CASTEP. To speed up our calculations, the symmetry was restricted to $P 4 / \mathrm{mmm}$.

Each unit cell of the infinite chain contained one atom, with the chain oriented along the $z$ axis $(c \sim 2.5 \AA)$, and, to avoid interactions between periodic images, at least $10 \AA$ vacuum in the $\hat{x}$ and $\hat{y}$ directions $(a=b=10 \AA)$ [31]. We first optimized the interatomic separation between the individual atoms in the chain by varying the cell size along $z$, while keeping all other dimensions fixed, until the per-atom force fell below $10^{-2} \mathrm{eV} / \AA$. We used the TPSD algorithm [32] for constrained relaxations. Then, starting from the equilibrium separation, we increased the interatomic spacing within the chains, incrementing $c$ by $0.1 \AA$ at a time, and calculated the total energy as a function of interatomic separation, similar to Ref. [31]. Figure 4(a) shows the results of these calculations.

The total energy in Fig. 4(a) clearly rises more steeply with interatomic separation in the case of relativistic gold 

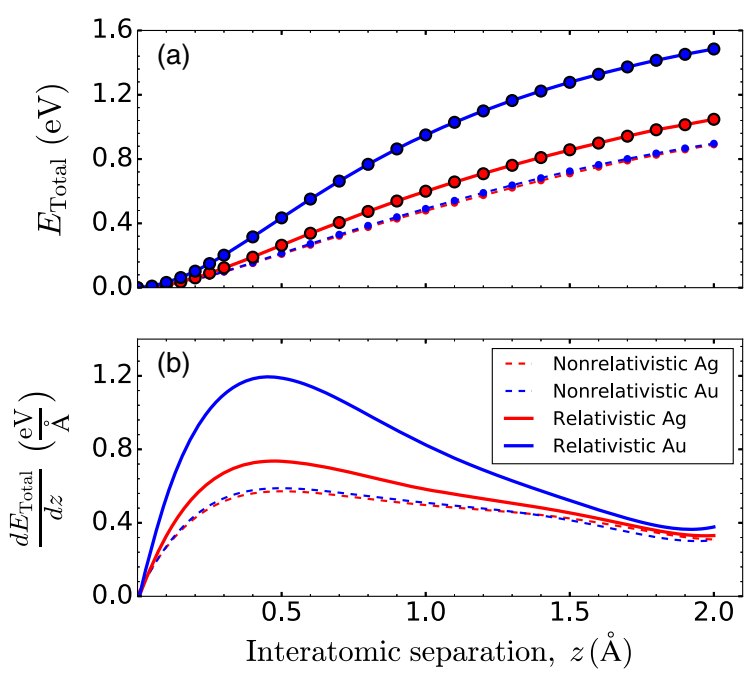

FIG. 4. (Rescaled) total energy (a), and its derivative (b), as a function of (rescaled) interatomic separation $z$ between atoms in infinite monatomic chains of nonrelativistic gold (dashed blue) and silver (dashed red), and scalar relativistic gold (solid blue) and silver (solid red). The derivative curves in (b) were obtained from a fit of the total-energy data in (a) to sixth-order polynomials. In the absence of scalar relativistic corrections, monatomic gold and silver chains exhibit almost identical force curves. Conversely, the interaction "force" between relativistic gold atoms in (b) rises, from large separations, to the maximum value for relativistic silver atoms a distance $\sim 0.7 \AA$ earlier.

(in the figure, the origin coincides with the equilibrium interatomic separations and corresponding energies of the chains). To extract parameters that help us to better characterize these differences, we fit the data to a Rose's universal binding curve [33], $U=-\alpha\left(x-x_{0}\right) e^{-\beta\left(x-x_{0}\right)}+E_{0}$. This model relates the equilibrium bond length $x_{\mathrm{eq}}=x_{0}+$ $1 / \beta$ and energy $E_{\mathrm{eq}}=E_{0}-\alpha / e \beta$ to a number of fitting parameters of physical importance, such as the breaking force $F_{\text {break }}=-\alpha / e^{2}$ and interelectrode binding constant $k_{\text {bind }}=-\alpha \beta / e^{3}$. Table II records the fits of our DFT total

TABLE II. Results of fitting DFT total energy data to Rose's universal binding potential [33]. The "rel." stands for relativistic effects included and "no rel." for the case when relativistic effects are not included in the simulations.

\begin{tabular}{lcccc}
\hline \hline & Au no rel. & Au rel. & Ag no rel. & Ag rel. \\
\hline$\alpha(\mathrm{eV} / \AA)$ & 4.48 & 8.66 & 4.34 & 5.46 \\
$\beta(1 / \AA)$ & 1.58 & 1.99 & 1.45 & 1.66 \\
$x_{0}(\AA)$ & 2.2 & 2.1 & 2.05 & 2.052 \\
$E_{0}(\mathrm{eV})$ & -14080 & -14130 & -3970 & -4004 \\
$x_{\text {eq }}(\AA)$ & 2.86 & 2.58 & 2.74 & 2.65 \\
$E_{\text {eq }}(\mathrm{eV})$ & -1.084 & -1.60 & -1.10 & -1.21 \\
$x_{\text {break }}(\AA)$ & 3.52 & 3.09 & 3.43 & 3.26 \\
$F_{\text {break }}(\mathrm{eV} / \AA)$ & -0.61 & -1.17 & -0.59 & -0.74 \\
$x_{\text {bind }}(\AA)$ & 4.18 & 3.59 & 4.12 & 3.86 \\
$k_{\text {bind }}\left(\mathrm{eV} / \AA^{2}\right)$ & -0.38 & -0.86 & -0.31 & -0.45 \\
\hline \hline
\end{tabular}

energy data to this model. We note that the theoretical equilibrium interatomic separation of relativistic gold, $2.58 \AA$, agrees well with the experimental range of values, $2.5 \pm 0.2 \AA$, reported in Ref. [34].

We observe that the breaking force is twice as high when relativistic effects are included. The obtained values, $\sim 1.9$ and $1.2 \mathrm{nN}$ for relativistic $\mathrm{Au}$ and $\mathrm{Ag}$, respectively, exhibit good agreement with experiment and previous calculations [17,22]. The maxima of the derivative curves in Fig. 4(b) can be identified with the "force" required to break the monatomic chains $[9,31]$. It is remarkable that the relativistic gold atoms already experience an interaction force, equal in magnitude to the maximum force between relativistic silver atoms, when the separation between them is $\sim 0.7 \AA$ greater. In particular, in the absence of scalar relativistic corrections, the force curves are virtually identical. In reality, there is a small horizontal offset $(\sim 0.1 \AA$, not shown) between these two force curves, due to their slightly different equilibrium chain lengths. The origin of the much stronger interaction between relativistic gold atoms can be traced to the significant radial contraction of their outer $6 s$ orbitals, which is not seen in the corresponding orbitals of the other three cases, due to the relativistic mass effect [1]. As a result, the gold atoms experience a relatively deeper potential well, and hence steeper energy gradient, within the monatomic chain. Furthermore, the radial expansion of the outer $5 d$ and $4 f$ orbitals in $\mathrm{Au}$, resulting from the stronger screening of the nucleus by radially contracted $s$ and $p$ orbitals at lower energy levels [1], may lead to a much higher density of states at the Fermi level, contributed by the $5 d$ orbitals [35], and, thus, to a stronger interelectrode interaction.

The above considerations can be accounted for by the effective spring constant $k_{\text {bind }}$ of the interelectrode potential. The occurrence of jump to contact has been modeled in the literature as resulting from a competition between intraand interelectrode potentials, represented by their respective elastic constants ( $k_{\text {inter }}$ and $\left.k_{\text {intra }}\right)$ : a jump to contact occurs when $k_{\text {inter }} / k_{\text {intra }}>1$, with a larger ratio, resulting in a larger jump [17]. The calculated binding potential in Fig. 4 is a reasonable estimate of the interelectrode potential between the last two atoms of the junction, so that $k_{\text {inter }}$ can then be approximated by the extracted values of $k_{\text {bind }}$ in Table II. In the literature, the intraelectrode potential is often modeled by a spring potential [17], where $k_{\text {intra }}$ is then represented by the spring constant $k_{\mathrm{el}}$. For realistic electrodes the geometry, that is, the atomic coordination, determines this intraelectrode elastic constant [17]. One may then assume that $k_{\mathrm{el}}$ would be similar for identical $\mathrm{Ag}$ and $\mathrm{Au}$ electrode geometries; the size of the jump will then mainly be determined by the interelectrode constant $k_{\text {bind }}$. Noting that the interelectrode binding constant $k_{\text {bind }}$ of relativistic Au is double that of all the other cases and taking the intraelectrode elasticity arguments presented above into account, it is expected that relativistic 
Au will exhibit a larger jump to contact. This is precisely what we observe in our experimental data.

In summary, we have reported a direct measurement of the strong relativistic effect in the formation of single-atom gold contacts. This phenomenon was revealed by the introduction of a new statistical treatment of the experimental data, and can be fully understood from a comparison of the experiments with DFT calculations in which scalar relativistic corrections are included or not.

This work has been funded by the Spanish Government through Grants No. FIS2013-47328 and No. MAT201678625 and the Conselleria d'Educaci, Investigaci, Cultura i Esport de la Generalitat Valenciana, PROMETEO/2017/ 139. C. S. gratefully acknowledges financial support from SEPE Servicio Público de Empleo Estatal. W. D. acknowledges funding from the National Research Foundation of South Africa through the Innovation Doctoral scholarship programme, Grant No. UID 102574. W. D. also thanks Professor A. E. Botha for sharing the PYTHON scripts used in the polynomial interpolations and E. Artacho, J. Fernández-Rossier, and J. J. Palacios for fruitful discussions. The DFT calculations in this paper were performed on the high-performance computing (HPC) facility at UNISA.

*r.calvo@nanogune.eu

†Present address: Chemical Physics Department, Weizmann Institute of Science, 76100 Rehovot, Israel. *untiedt@ua.es

[1] P. Pyykko, Chem. Rev. 88, 563 (1988).

[2] F. Calvo, E. Pahl, M. Wormit, and P. Schwerdtfeger, Angew. Chem., Int. Ed. Engl. 52, 7583 (2013).

[3] K. M. Ho and K. P. Bohnen, Phys. Rev. Lett. 59, 1833 (1987).

[4] D. J. Gorin and F. D. Toste, Nature (London) 446, 395 (2007).

[5] N. Takeuchi, C. T. Chan, and K. M. Ho, Phys. Rev. Lett. 63, 1273 (1989).

[6] A. Filippetti and V. Fiorentini, Surf. Sci. 377, 112 (1997).

[7] R. H. M. Smit, C. Untiedt, A. I. Yanson, and J. M. van Ruitenbeek, Phys. Rev. Lett. 87, 266102 (2001).

[8] H. Häkkinen, R. N. Barnett, A. G. Scherbakov, and U. Landman, J. Phys. Chem. B 104, 9063 (2000).

[9] S. R. Bahn and K. W. Jacobsen, Phys. Rev. Lett. 87, 266101 (2001).

[10] A. Hasmy, L. Rincón, R. Hernández, V. Mujica, M. Márquez, and C. González, Phys. Rev. B 78, 115409 (2008).

[11] A. Thiess, Y. Mokrousov, S. Blugel, and S. Heinze, Nano Lett. 8, 2144 (2008).
[12] N. Agraït, A. L. Yeyati, and J. M. Van Ruitenbeek, Phys. Rep. 377, 81 (2003).

[13] J. Kröger, N. Néel, A. Sperl, Y. F. Wang, and R. Berndt, New J. Phys. 11, 125006 (2009).

[14] C. Untiedt, M. J. Caturla, M. R. Calvo, J. J. Palacios, R. C. Segers, and J. M. van Ruitenbeek, Phys. Rev. Lett. 98, 206801 (2007).

[15] C. Sabater, M. J. Caturla, J. J. Palacios, and C. Untiedt, Nanoscale Res. Lett. 8, 257 (2013).

[16] L. Limot, J. Kröger, R. Berndt, A. Garcia-Lekue, and W. A. Hofer, Phys. Rev. Lett. 94, 126102 (2005).

[17] M. L. Trouwborst, E. H. Huisman, F. L. Bakker, S. J. van der Molen, and B. J. van Wees, Phys. Rev. Lett. 100, 175502 (2008).

[18] M. A. Fernández, C. Sabater, W. Dednam, J. J. Palacios, M. R. Calvo, C. Untiedt, and M. J. Caturla, Phys. Rev. B 93, 085437 (2016).

[19] E. Scheer, N. Agraït, J. C. Cuevas, A. L. Yeyati, B. Ludoph, A. Martín-Rodero, G. R. Bollinger, J. M. van Ruitenbeek, and C. Urbina, Nature (London) 394, 154 (1998).

[20] log denotes here the common logarithm (base 10).

[21] C. Sabater, W. Dednam, M. R. Calvo, M. A. Fernández, C. Untiedt, and M. J. Caturla, Phys. Rev. B 97, 075418 (2018).

[22] M. S. Hybertsen and L. Venkataraman, Acc. Chem. Res. 49, 452 (2016).

[23] S. J. Clark, M. D. Segall, C. J. Pickard, P. J. Hasnip, M. I. Probert, K. Refson, and M. C. Payne, Z. Kristallogr. 220, 567 (2005).

[24] D. Vanderbilt, Phys. Rev. B 41, 7892 (1990).

[25] K. Lejaeghere, V. Van Speybroeck, G. Van Oost, and S. Cottenier, Crit. Rev. Solid State Mater. Sci. 39, 1 (2013).

[26] D. D. Koelling and B. N. Harmon, J. Phys. C 10, 3107 (1977).

[27] F. Strigl, C. Espy, M. Bückle, E. Scheer, and T. Pietsch, Nat. Commun. 6, 6172 (2015).

[28] J. P. Perdew, K. Burke, and M. Ernzerhof, Phys. Rev. Lett. 77, 3865 (1996).

[29] A. Tkatchenko and M. Scheffler, Phys. Rev. Lett. 102, 073005 (2009).

[30] A. Ambrosetti and P. L. Silvestrelli, Phys. Rev. B 94, 045124 (2016).

[31] M. S. Hybertsen, J. Chem. Phys. 146, 092323 (2017).

[32] J. Barzilai and J. M. Borwein, IMA J. Numer. Anal. 8, 141 (1988).

[33] J. H. Rose, J. Ferrante, and J. R. Smith, Phys. Rev. Lett. 47, 675 (1981).

[34] C. Untiedt, A. I. Yanson, R. Grande, G. Rubio-Bollinger, N. Agraiit, S. Vieira, and J. M. van Ruitenbeek, Phys. Rev. B 66, 085418 (2002).

[35] O. Adak, R. Korytár, A. Y. Joe, F. Evers, and L. Venkataraman, Nano Lett. 15, 3716 (2015). 